\title{
FEEDBACK-EFFICIENT AGILE SCHEDULING-BEAMFORMING FOR NETWORK MIMO OFDMA SYSTEMS WITH REALISTIC CHANNEL MODELS
}

\author{
Behrouz Khoshnevis, Wei Yu \\ ECE Dept., University of Toronto \\ \{bkhoshnevis, weiyu\}@comm.utoronto.ca
}

\author{
Yves Lostanlen \\ Siradel Canada \\ YLostanlen@siradel.com
}

\begin{abstract}
The acquisition of channel state information is a pressing issue in the implementation of network multi-input multi-output (MIMO) systems, where the base-stations coordinate across multiple cells for intercell interference mitigation. This paper proposes a two-stage channel quantization and feedback mechanism named agile scheduling-beamforming (ASB) for the downlink network MIMO system employing both spatial multiplexing and orthogonal frequency-division multipleaccess (OFDMA). The proposed scheme polls the users for their best set of spatial-frequency resource blocks, then schedules the users according to the fairness criterion, and finally asks the selected users to feedback finer channel quantization. This paper utilizes insights derived from the scaling law of the optimal quantization bit allocation for this scheme, and evaluates its performance on realistic channel models. Rate map simulations based on ray-tracing-based wireless propagation models of realistic urban small-cell deployment show that the proposed scheme can already approach the performance of network MIMO with full channel state information with only modest amount of channel feedback.
\end{abstract}

\section{INTRODUCTION}

Network multi-input multi-output (MIMO), also known as coordinated multipoint (CoMP), is a promising future architecture for wireless cellular systems with densely deployed base-stations. By coordinating multiple cells via high-speed backhaul, a downlink network MIMO system can jointly transmit to remote users across the multiple base-stations, thus completely eliminating intercell interference. The implementation of network MIMO systems, however, is also challenging due to its high demand on the network-wide channel state information (CSI) aquisition at the user terminals. Compounding this with the fact that the scheduling step for a network MIMO system involves a larger pool of potential users, and that the CSI of all these users needs to be fed back for scheduling purposes, a conventional implementation of a channel quantization feedback scheme can quickly overwhelm the benefit of network MIMO.

This paper proposes the use of a two-stage channel quan- tization and feedback scheme that is capable of significantly reducing the CSI feedback requirement in a network MIMO system. The proposed scheme is named agile schedulingbeamforming (ASB), designed for a network MIMO system employing spatial multiplexing with orthogonal frequency division multiple access (OFDMA). In the first stage of the proposed scheme, coarse channel information is acquired from a large pool of users by the feedback of each user's best set of spatial-frequency resource blocks for scheduling. The subsequent second stage acquires finer CSI from the scheduled users for the purpose of designing network beamformers.

The proposed channel feedback mechanism is an extension of our previous work [1] on the scaling laws of optimal quantization bit allocation for the network MIMO systems, but with two additional elements. First, this paper considers a combined spatial multiplexing and OFDMA setting in which the users are scheduled across the spatial and frequency dimensions. Second, a proportional fairness scheduler is incorporated to allow the maximization of network utility. Further, this paper makes design choices under practical deployment scenarios, and evaluates the overall system using realistic raytracing-based wireless propagation models in actual urban environments [2]. These accurate models enable the generation of rate maps that show the impact of limited feedback on practical network MIMO deployment.

Limited-feedback schemes for multiuser MIMO systems have been studied extensively in the literature. For example, $[3,4]$ proposed a scheme in which a library of orthogonal beamforming codebooks is constructed at the base-station; each user reports its best beamformers across the codebooks; the best orthogonal codebook among all codebooks in the library is then chosen. Like the scheme in $[3,4]$, the present paper also utilizes a fixed set of orthogonal beamformers in the first stage. However, this paper suggests that a subsequent second stage that refines the channel quantization can significantly improve the overall performance. The idea of two-stage beamforming and scheduling has been previously considered in [5], but the first stage feedback proposed in [5] consists of a coarse channel quantization by every user, rather than the more efficient scheme proposed here where each user reports only the best set of spatial-frequency resource blocks. 
In the OFDMA context, this paper treats the frequency resource the same way as the spatial resource, and applies the two-stage scheme to the entire spatial-frequency grid. When restricted to OFDMA systems, the first stage of the approach proposed in this paper is similar to that in [6]. The approach of this paper is different and is easier to implement than the contention and threshold-based schemes of $[7,8]$ in which the users vie for the scheduling slots, because the creation of a contention channel is not necessarily straightforward. There are also many works in the literature considering the interpolation, clustering, and grouping of beamformers across the OFDM tones $[9,10,11]$. Some of these techniques can be applied on top of the schemes proposed here.

Finally, this paper considers the problem of channel quantization only. Channel estimation error and the issue of channel feedback delay [12] are not considered for simplicity.

\section{SYSTEM MODEL}

Consider a downlink network MIMO system consisting of $I$ base-stations, each with $M$ antennas, cooperating in transmitting data to a total of $U$ single-antenna users. The basestations share users' data and channel state information (CSI) in order to collectively make scheduling decisions and perform downlink beamforming. To provide CSI, the users quantize and feedback their channel information through a ratelimited control channel. In this paper, the total feedback rate is assumed to be limited by $B$ bits per coherence time.

The system uses OFDM transmission, in which the total system bandwidth is divided into sub-carriers. Further, sub-carriers within the coherence bandwidth are grouped into physical resource blocks (PRB). To reduce the channel feedback rate, every $K$ adjacent PRBs are further grouped into what we call a sub-band. Assuming that there are a total number of $N$ PRBs, this results in $N_{s}=N / K$ sub-bands in the system. The sub-band size determines the resolution of the users' CSI feedback over the frequency domain.

Performing optimal scheduling and beamforming would require perfect CSI feedback from all the users in both the spatial and the frequency dimensions. The main contribution of this paper is an efficient way of feeding back this twodimensional CSI in a limited-feedback system using a novel algorithm referred to as agile scheduling-beamforming.

\section{LIMITED-FEEDBACK NETWORK MIMO}

The fundamental problem in the design of limited-feedback multiuser system is the following. In a limited-feedback system involving many users, on one hand a large number of users need to feedback their CSI for scheduling purpose in order to benefit from multiuser diversity. On the other hand, only a small number of users are eventually scheduled; hence accurate CSI is needed only from a few. For this reason, it is fundamentally inefficient to design a feedback system in

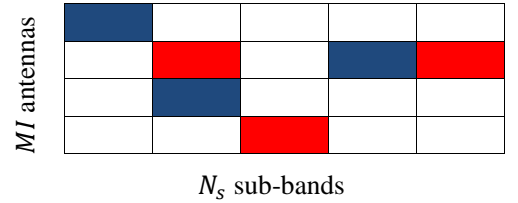

Fig. 1. CSI matrix. Two different colors show the best three (antenna,sub-band) pairs for two different users.

which equal number of feedback bits are assigned to each user. This observation is first made for single-cell multiuser MIMO systems [13], but is equally applicable in the network MIMO context. Motivated by this observation, this paper proposes a two-stage feedback scheme in which coarse CSI is collected from many users for scheduling purpose, while accurate CSI is collected from only a few for spatial multiplexing. The proposed CSI feedback mechanism and schedulingbeamforming procedure are described below.

\subsection{First-Stage CSI Feedback}

The goal of first-stage CSI feedback is to obtain a coarse quantization of the channels from a large number of users for scheduling purpose. However, instead of directly employing a coarse quantizer (as in [5]), this paper adopts a more efficient mechanism in which a set of orthogonal beamforming vectors are fixed across the entire network MIMO system, then each user reports its best set of sub-channels over all spatial and frequency dimensions. The initial set of orthogonal beamformers can be arbitrary. In fact, a simple identity matrix can serve as the initial beamforming matrix, in which case each user's spatial-frequency CSI is simply a two-dimensional matrix consisting of the channel responses from each of the antennas at each of the base-stations on each of the sub-bands. This is an $M I \times N_{s}$ matrix as shown in Fig. 1 .

To efficiently send a coarse quantization of each user's sub-channels to the base-stations, we propose a first-stage quantization mechanism in which $U$ users in the network are asked to rank their spatial-frequency sub-channels in the CSI matrix according to some scheduling criterion, then send back the indices and some measure of qualities of the $S$ best subchannels. (For simplicity, the rest of this paper assumes that the channel magnitudes are fed back perfectly. This is justified by the fact that the number of bits required for the quantization of the channel quality is usually smaller than the number of bits needed for feeding back the indices of the best $S$ subchannels, especially when the number of sub-bands is large.) For example, Fig. 1 shows the best $S=3$ entries for two different users marked by different colors. With this setup, the first stage consumes

$$
B_{1}=U S \log _{2}\left(M I N_{s}\right) \quad \text { bits }
$$

for feeding back the sub-channel indices. This information is used for the scheduling of users on the available spatial- 


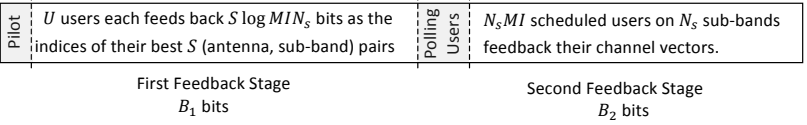

Fig. 2. Two-stage CSI feedback scheme

frequency resource blocks. The scheduling procedure also takes fairness and user priorities into account.

\subsection{Second-Stage CSI Feedback}

After the users are scheduled based on the first-stage information, the scheduled users are polled for the quantized values of their channel vectors from all the base-stations in the second stage. For this purpose, the total number of bits available for the second stage, $B_{2}=B-B_{1}$, is divided equally among the $N_{s}$ sub-bands. The $B_{2} / N_{s}$ bits on each sub-band is then divided equally among the users scheduled on that sub-band. For clarity, assume an arbitrary sub-band with $U_{s}$ users scheduled on it (note that $\left|U_{s}\right|<M I$ as we have a total of $M I$ antennas on each sub-band). Each user on this sub-band can therefore use a total of $b=B_{2} /\left(N_{s} U_{s}\right)$ bits for quantizing its $I$ channel vectors from each base-station, each of which is an $M \times 1$ complex vector.

To complete the specifications of the second stage, we need to determine how each user allocates its $b$ bits for quantizing the $I$ channel vectors from different base-stations. For this purpose, we use the bit allocation rule in [1], where the quantization bits are allocated in proportion to the norm of the channel vector in logarithmic scale. This approach is shown to minimize the residual interference caused by the inevitable inaccuracy of the beamforming vector design due to limited CSI. The two feedback stages are summarized in Fig. 2.

\subsection{Agile Scheduling-Beamforming}

This paper proposes an agile scheduling-beamforming algorithm for efficiently utilizing the two-stage feedback mechanism. The scheduling procedure together with the first-stage feedback is now described in more detail. Most practical scheduling algorithm is based on selecting the user with the maximum weighted achievable rate, where the weights are chosen according to some higher level protocols. For example, in a proportional fairness scheduler, the weights are set to be reciprocal of each user's respective long-term average rate. Thus, an efficient use of the first-stage feedback is to let each user rank sub-channels in the CSI matrix according to an estimate of the weighted rate.

Let $h_{m i s u}$ denote the scalar channel from antenna $m$ on base-station $i$ to user $u$ on sub-band $s$. This paper proposes to use the following quantity as an estimate of weighted rate for the (antenna, sub-band) pair $(m i, s)$ :

$$
\gamma_{\text {misu }}=w_{u} \log \left(1+\frac{\rho\left|h_{\text {misu }}\right|^{2}}{1+\rho \sum_{(n j) \neq(m i)}\left|h_{n j s u}\right|^{2}}\right)
$$

where $w_{u}$ is the weight for user $u$ and $\rho$ is the transmit SNR. In essense, this surrogate rate expression assumes that useful information is carried only on one antenna, and all other antennas contribute to the interference. However imperfect such an estimate of the weighted rate may be, it nonetheless gives some relative measure of the desirability of scheduling a particular user on the sub-channel in question. Given that $h_{m i s u}$ is the only information each user has access to, this choice of channel quality measure is reasonable.

With the surrogate rate defined in (2), the scheduling operation can now be carried out as follows:

$$
\pi(m i, s)=\arg \max _{u \in \mathcal{U}_{m i, s}} \gamma_{m i s u}
$$

where $\mathcal{U}_{m i, s}$ is the set of users who have reported on the (antenna, sub-band) pair $(m i, s)$.

After the scheduling procedure is completed, the CSI of the scheduled users is collected in the second feedback stage. This information is then used to design the zero-forcing beamforming vectors for the scheduled users by assuming that the quantized channel vectors in the second stage are accurate.

\subsection{Scaling Laws and Practical Design Choices}

The two-stage feedback and agile scheduling-beamforming scheme proposed in this paper is an extension of our earlier work [1] where the scaling laws of two-stage channel feedback is studied. Assuming a system with a large number of users (but without OFDMA or proportional fairness scheduling), it is concluded in [1] that to achieve both multiuser diversity gain and spatial multiplexing gain:

- Most of the feedback bits should be devoted to the first stage. In fact among the total $B$ feedback bits, the second stage should only use $O(\log (B))$ bits;

- In the second stage, the allocation of quantization bits among the different vector channels should be in proportion to the channel gain in $\mathrm{dB}$ scale; (see also [12]).

The algorithm proposed in the present paper already incorporates the second insight above. But for the optimal allocation of feedback bits in the first stage, the number of users in a practical deployment is typically smaller than the asymptotic regime of [1]. The simulation of the next section makes practical design choices based on realistic setups. In this practical case, we actually allocate more bits for the second stage.

\section{SIMULATIONS}

The effectiveness of two-stage channel quantization feedback and agile scheduling-beamforming is first evaluated using a 


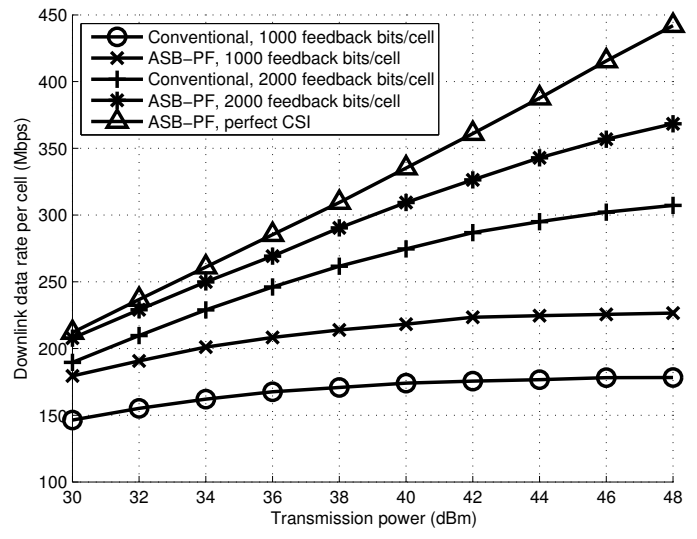

Fig. 3. Sum rate vs transmit power

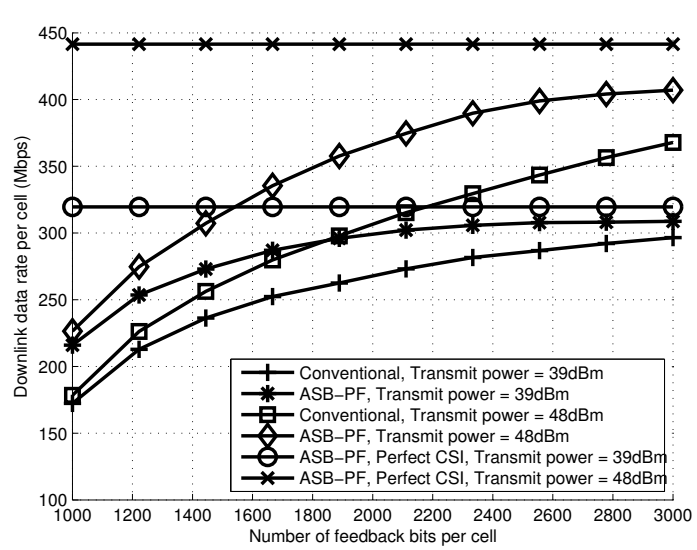

Fig. 4. Sum rate vs number of feedback bits

wireless propagation model with a path-loss exponent of 3.8 and with Rayleigh fading for a macrocell network MIMO deployment with 7 cells and 30 users per cell over $20 \mathrm{MHz}$ bandwidth. The base-stations are equipped with two antennas each, and are $1 \mathrm{~km}$ apart. Figs. 3 and 4 show the achievable downlink sum rate per cell as a function of total number of feedback bits per cell and transmit power. Fig. 5 shows the cumulative density function of the achieved user rates under proportionally fair scheduling. The conventional scheme here refers to the scheme in which each user quantizes its channel with a fixed number of bits. The perfect CSI scheme refers to the scheme in which perfect CSI is known in both stages of the two-stage ASB process.

It is clear that the two-stage ASB algorithm significantly outperforms conventional feedback. With 3000 feedback bits per cell per coherence time and the transmit power of $39 \mathrm{dBm}$ per base-station (as shown in Fig. 4), the network MIMO with imperfect CSI can already approach the perfect CSI case.

To further validate the simulation results, the two-stage ASB algorithm is tested on a realistic ray-tracing-based model

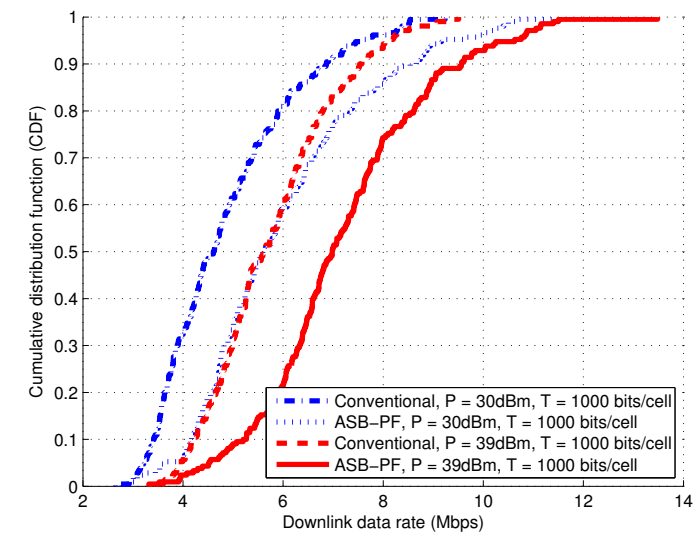

Fig. 5. Cumulative density function of user rates

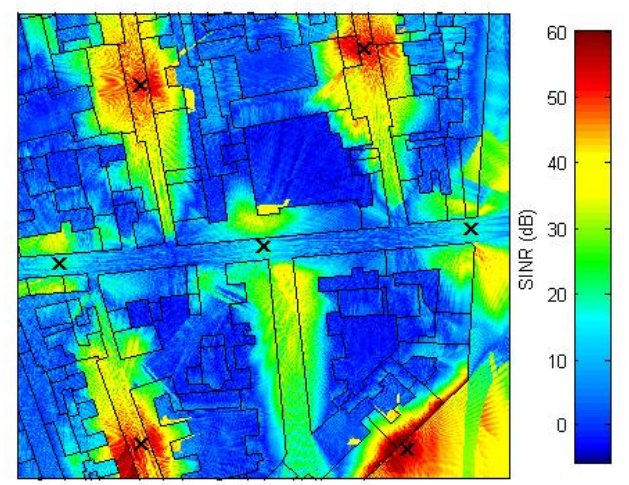

Fig. 6. SINR map of a small-cell deployment in Paris.

[2] of an urban small-cell deployment with 7 base-stations with two antennas each and 7 users per cell over an $240 \mathrm{~m}$ by $240 \mathrm{~m}$ area. The carrier frequency is at $763 \mathrm{MHz}$; the bandwidth is $10 \mathrm{MHz}$; the transmit power is $20 \mathrm{dBm}$. As shown in the SINR map in Fig. 6, without network MIMO only the areas with a line of sight from the base-station get appreciable SINR, whereas much of elsewhere experiences poor SINR.

The situation improves dramatically with network MIMO. As shown in Fig. 7, with 8000 feedback bits per cell, most of the areas in the cell are now capable of supporting $10-20 \mathrm{Mbps}$ per user. In fact, with perfect CSI, the simulated per-cell sum rate is about $245 \mathrm{Mbps}$, and the two-stage ASB algorithm already achieves $88 \%$ of the perfect CSI rate with only 8000 feedback bits. If we assume a coherence time of $1 \mathrm{~ms}$ (roughly corresponding to vehicular speed), the total two-stage feedback overhead is well below $10 \%$ of the downlink total rate. This particular ASB scheme is optimized with sub-band spacing of about $180 \mathrm{KHz}$, and where each user feeds back its best $S=21$ spatial-frequency sub-channels. About $20 \%$ of the total feedback bits are devoted to the first stage in this case. 

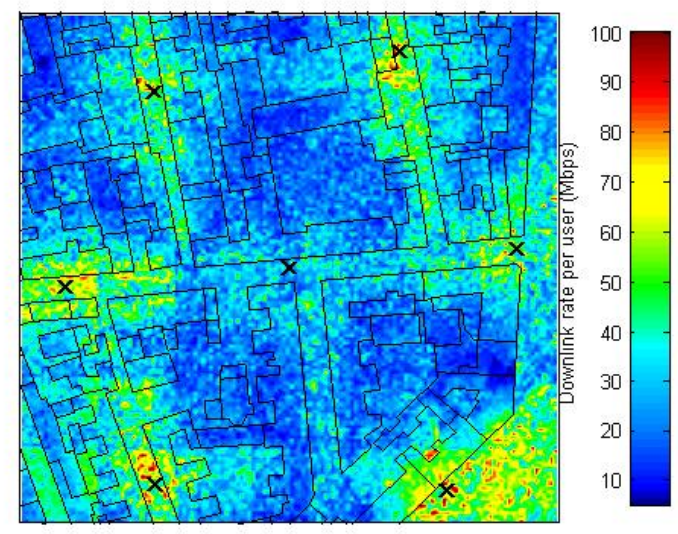

Fig. 7. Network MIMO rate map with limited feedback
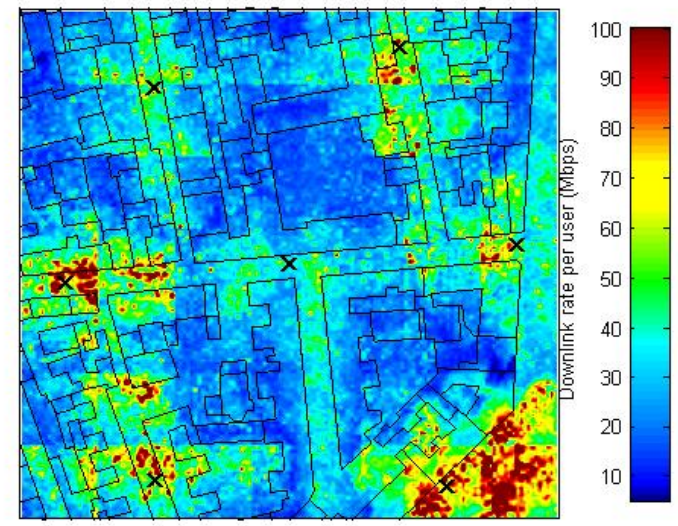

Fig. 8. Network MIMO rate map with perfect CSI

\section{CONCLUSION}

This paper shows that it is possible to design efficient CSI feedback scheme for the network MIMO system to approach the perfect CSI performance with modest amount of feedback. The key idea is to use a two-stage feedback mechanism together with an agile scheduling and beamforming scheme to capture the benefit of multiuser diversity in the first stage and that of spatial multiplexing gains in the second stage.

\section{REFERENCES}

[1] B. Khoshnevis and W. Yu, "Two-stage channel feedback for beamforming and scheduling in network MIMO systems," in IEEE Int. Conf. Commun. (ICC), June 2011.

[2] Y. Corre and Y. Lostanlen, "Three-dimensional urban EM wave propagation model for radio network planning and optimization over large areas," IEEE Trans. Veh. Technol., vol. 58, no. 7, pp. 3112-3123, Sept. 2009.

[3] K. Huang, R. W. Heath, and J.G . Andrews, "Space division multiple access with a sum feedback rate constraint," IEEE Trans. Signal Process., vol. 55, no. 7, pp. 3879-3891, July 2007.

[4] K. Huang, J. G. Andrews, and R. W. Heath, "Performance of orthogonal beamforming for SDMA with limited feedback," IEEE Trans. Veh. Technol., vol. 58, no. 1, pp. 152-164, Jan. 2009.

[5] R. Zakhour and D. Gesbert, "A two-stage approach to feedback design in multi-user MIMO channels with limited channel state information," in IEEE 18th Int. Symp. Personal, Indoor and Mobile Radio Commun. (PIMRC), Sept. 2007, pp. 1-5.

[6] J. Leinonen, J. Hamalainen, and M. Juntti, "Performance analysis of downlink OFDMA resource allocation with limited feedback," IEEE Trans. Wireless Commun., vol. 8, no. 6, pp. 2927-2937, June 2009.

[7] R. Agarwal, V. Majjigi, Z. Han, R. Vannithamby, and J. Cioffi, "Low complexity resource allocation with opportunistic feedback over downlink OFDMA networks," IEEE J. Sel. Areas Commun., vol. 26, no. 8, pp. 14621472, Oct. 2008.

[8] T. Tang, R. W. Heath, S. Cho, and S. Yun, "Opportunistic feedback for multiuser MIMO systems with linear receivers," IEEE Trans. Commun., vol. 55, no. 5, pp. 1020-1032, May 2007.

[9] J. Choi and R. W. Heath, "Interpolation based transmit beamforming for MIMO-OFDM with limited feedback," IEEE Trans. Commun., vol. 53, no. 11, pp. 41254135, Nov. 2005.

[10] T. Pande, D. J. Love, and J. V. Krogmeier, "Reduced feedback MIMO-OFDM precoding and antenna selection," IEEE Trans. Signal Process., vol. 55, no. 5, pp. 2284-2293, May 2007.

[11] J. Chen, R. Berry, and M. Honig, "Limited feedback schemes for downlink OFDMA based on sub-channel groups," IEEE J. Sel. Areas Commun., vol. 26, no. 8, pp. 1451-1461, Oct. 2008.

[12] R. Bhagavatula and R. W. Heath, "Adaptive bit partitioning for multicell intercell interference nulling with delayed limited feedback," IEEE Trans. Signal Process., vol. 59, no. 8, pp. 3824-3836, Aug. 2011.

[13] N. Ravindran and N. Jindal, "Multi-user diversity vs. accurate channel feedback for MIMO broadcast channels," in IEEE Int. Conf. Commun. (ICC), May 2008, pp. 3684-3688. 